\title{
Properties of Composition of Fuzzy Relations and its Verifications
}

\author{
C. Gowrishankar, R. Dharshinee, K. Geetha
}

\begin{abstract}
This paper, deals with fundamental notions in pure and applied sciences, i.e., basic operations related to fuzzy relations. The composition of fuzzy relations are defined in two ways such as max-min composition and max-product composition with suitable example. This paper also introduces the properties of composition of fuzzy relations. The newly introduced properties inculcates zero, identity, equal, not-equal, subset, associative, union, intersection and distributive fuzzy relations. Finally, the paper verifies the properties of composition of fuzzy relations using some numerical values for $2 \times 2$ order of matrix. Also gives some exercise problems related to the above concepts with accurate answer keys.
\end{abstract}

Keywords: Fuzzy sets, Fuzzy relations, Cartesian product, Union, Intersection, Composition of fuzzy relations

\section{INTRODUCTION}

Fuzzy relation was introduced by L.A.Zadeh in the year 1965. In general, fuzzy relations are the concept of relations in the same manner as fuzzy sets generalize the fundamental idea of sets.

A classical set theory is defined by crisp(exact) boundaries. i.e.,there is no uncertainty about the location of the set boundaries and widely used in digital system design. For example, the question arise "Is water colorless?" then the only crisp sets are "Yes or No". Therefore, classical set theory allows the membership of the elements in the set in binary terms.

The word "fuzzy" means "vagueness or ambiguity", i.e., fuzziness occurs when the boundary of a piece of information is not clear-cut. For example, the words like young, tall, good or high are fuzzy sets. In above examples the term young defines there is no single quantitative value (i.e., for some people age 25 is young and for others age 35 is young). Therefore, the concept young has no clean boundary.

Also, fuzzy set theory is an extension of classical set theory which is defined for research approach that can deal with problems relating to ambiguous boundaries. i.e., there exists uncertainty about the location of the set boundaries and which is used in fuzzy controllers. For example, the statement arise "Is Ravi Honest" then the fuzzy sets are, "Extremely Honest (1), Very Honest(0.90), Honest at time (0.50) and Extremely Dishonest (0.0)".

Revised Manuscript Received on January 03, 2020.

* Correspondence Author

C. Gowrishankar, Department of Mathematics, Government Arts and Science College, Gudalur-643 212, Tamil Nadu, India. E-mail: cgowrishankar88@gmail.com

R. Dharshinee, Department of Mathematics, Government Arts and Science College, Gudalur-643 212, Tamil Nadu, India.

K. Geetha, Department of Mathematics, Government Arts and Science College, Gudalur-643 212, Tamil Nadu, India.

(c) The Authors. Published by Blue Eyes Intelligence Engineering and Sciences Publication (BEIESP). This is an open access article under the CC BY-NC-ND license (http://creativecommons.org/licenses/by-nc-nd/4.0/)
Therefore, fuzzy set theory permits membership function valued in the interval $[0,1]$. Fuzzy relations are significant concepts in fuzzy theory and have been widely used in many fields such as fuzzy clustering, fuzzy control and uncertainty reasoning.

They also have used applications in some fields such as medicine, psychology, economics and sociology.

In that paper we discuss about the concept of composition of fuzzy relations and its properties with suitable examples. We have also given related exercise problems with answer keys.

\section{SOME BASIC DEFINITIONS OF FUZZY RELATIONS}

\subsection{Definition}

The Cartesian product $\tilde{A} \times \widetilde{B}=\{(x, y) / x \in \tilde{A}, y \in \widetilde{B}\}$. Where $\widetilde{A}$ and $\widetilde{B}$ are subsets of the universal sets $\mathrm{X}$ and $\mathrm{Y}$ correspondingly.

\subsection{Definition}

A fuzzy relation is a fuzzy set defined on the Cartesian product of crisp set $\mathrm{X}_{1}, \mathrm{X}_{2}, \ldots . \mathrm{X}_{\mathrm{n}}$, where tuples $\left(\mathrm{x}_{1}, \mathrm{x}_{2}, \ldots, \mathrm{x}_{\mathrm{n}}\right)$ that may have varying degrees of membership within the relation. The membership value is usually represented by a real number for the closed interval $[0,1]$ and indicates the strength of the present relation between elements of the tuple.

Consider $\widetilde{R}: X \mathrm{x} Y \rightarrow[0,1]$ then the fuzzy relation on $X \mathrm{x} Y$ denoted by $\tilde{R}$ or $\tilde{R}(x, y)$ is defined as the $\operatorname{set} \tilde{R}=\left\{(x, y), \mu_{\tilde{R}}(x, y) /(x, y) \in X \mathrm{x} Y\right\}$.

Where $\mu_{\tilde{R}}(x, y)$ is the strength of the relation in two variables called membership function.It gives the degree of membership of the ordered pair $(x, y)$ in $\widetilde{R}$ associating with each pair $(x, y)$ in $\mathrm{XxY}$ a real number in the interval $[0,1]$.

The degree of membership indicates the degree to which $x$ is in the relation with $y$.

\section{Example 2.1}

$x=\{3,5,8,10\}$ and

$X \times X=\{(3,3),(3,5),(3,8),(3,10), \ldots \ldots,(10,10)\}$ then $\mathrm{XRY}={ }^{\prime} x$ is considerably less than $\mathrm{y}^{\text {" }}$

$$
\tilde{R}=\mathrm{x}_{1} \mathrm{x}_{2}\left[\begin{array}{cccr}
\mathrm{x}_{1} & \mathrm{x}_{2} & \mathrm{x}_{3} & \mathrm{x}_{4} \\
\mathrm{x}_{3} & 0.5 & 0.8 & 1.0 \\
\mathrm{x}_{4} & 0 & 0.5 & 0.8 \\
0 & 0 & 0 & 0.3 \\
\mathrm{x}_{4} & 0 & 0 & 0
\end{array}\right]
$$

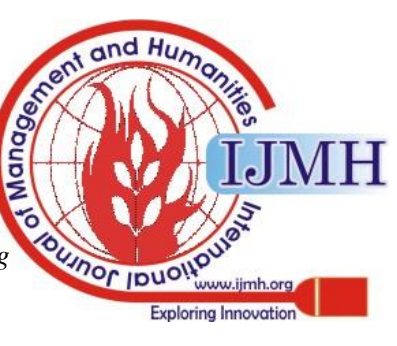




\section{Properties of Composition of Fuzzy Relations and its Verifications}

\subsection{Definition}

Let $\widetilde{A}$ and $\widetilde{B}$ be fuzzy sets on the universes Xand Y, then $\widetilde{A} \times \widetilde{B}=\widetilde{R} \subset \mathrm{XxY}$

Thenthe fuzzy relation $\tilde{R}$ has a membership function $\mu_{\tilde{R}}(x, y)=\mu_{\tilde{A} \mathrm{x} \tilde{B}}(x, \mathrm{y})=\min \left\{\mu_{\tilde{A}}(x), \mu_{\tilde{B}}(y)\right\}$ is called the fuzzy Cartesian product of XxY.

\section{Example 2.2}

Let $\tilde{A}$ defined on the universe of three discrete temperatures, $\mathrm{X}=\left\{x_{1}, x_{2}, x_{3}\right\}$, and $\tilde{B}$ defined on the universe of two discrete pressures, $\mathrm{Y}=\left\{y_{1}, y_{2}\right\}$. Fuzzy set $\tilde{A}$ represents the "ambient" temperature and fuzzy set $\widetilde{B}$ represents the "near optimum" pressure for a certain heat exchanger and the Cartesian product might represents the conditions (temperature-pressure pairs) of the exchanger that are associated with "efficient" operations.

For example, let $\tilde{A}=\frac{0.2}{x_{1}}+\frac{0.5}{x_{2}}+\frac{1}{x_{3}}$ and $\tilde{B}=\frac{0.3}{y_{1}}+\frac{0.8}{y_{2}}$ then

$$
\begin{array}{llll}
y_{1} & y_{2} & y_{1} & y_{2}
\end{array}
$$

$\tilde{A} \times \tilde{B}=\tilde{R}=\begin{gathered}x_{1} \\ x_{2} \\ x_{3}\end{gathered}\left[\begin{array}{cc}\min \{0.2,0.3\} & \min \{0.2,0.8\} \\ \min \{0.5,0.3\} & \min \{0.5,0.8\} \\ \min \{1,0.3\} & \min \{1,0.8\}\end{array}\right]=\begin{array}{r}x_{1} \\ x_{2} \\ x_{3}\end{array}\left[\begin{array}{cc}0.2 & 0.2 \\ 0.3 & 0.5 \\ 0.3 & 0.8\end{array}\right]$

\subsection{Definition}

The Cartesian product $\widetilde{A} \mathrm{x} \widetilde{B}$ is called a zero fuzzy relation, if $0=\left\{\left((x, y), \mu_{0}(x, y)\right) /(x, y) \in \tilde{A} \times \tilde{B}, \mu_{0}(x, y)=0\right\}$.

If we set $\mu_{\tilde{R}}(x, y)=1$ in the definition of fuzzy relation, we get classical relation.

\subsection{Definition}

The identity relation I defined for all $(x, y) \in \tilde{A} \mathrm{x} \widetilde{B}$ then its membership functions follows: $I=\mu_{I}(x, y)= \begin{cases}1, & \text { for } \mathrm{x}=\mathrm{y} \\ 0, & \text { for } \mathrm{x} \neq \mathrm{y}\end{cases}$

\section{Example 2.3}

The fuzzy relation

$$
\begin{aligned}
& \tilde{R}=\left\{\left(x_{1}, y_{1}\right), 0\right),\left(\left(x_{1}, y_{2}\right), 0.1\right),\left(\left(x_{1}, y_{3}\right), 0.2\right), \\
& \left(\left(x_{2}, y_{1}\right), 0.7\right),\left(\left(x_{2}, y_{2}\right), 0.2\right),\left(\left(x_{2}, y_{3}\right), 0.3\right),\left(\left(x_{3}, y_{1}\right), 1\right) \\
& \left.,\left(\left(x_{3}, y_{2}\right), 0.6\right),\left(\left(x_{3}, y_{3}\right), 0.2\right)\right\}
\end{aligned}
$$

Can also given by the table as follows:

$\tilde{R}=$\begin{tabular}{|c|c|c|c|}
\hline $\mathrm{Y}$ & $y_{1}$ & $y_{2}$ & $y_{3}$ \\
\hline$x_{1}$ & 0 & 0.1 & 0.2 \\
\hline$x_{2}$ & 0.7 & 0.2 & 0.3 \\
\hline$x_{3}$ & 1 & 0.6 & 0.2 \\
\hline
\end{tabular}

Find membership function.

\section{Solution:}

The numbers in the cells are located at the intersection of rows and columns. Then the values of the membership function as follows:

$\mu_{\tilde{R}}\left(\mathrm{x}_{1}, \mathrm{y}_{1}\right)=0, \mu_{\tilde{R}}\left(x_{1}, y_{2}\right)=0.1, \mu_{\tilde{R}}\left(\mathrm{x}_{1}, \mathrm{y}_{3}\right)=0.2$

$\mu_{\tilde{R}}\left(x_{2}, y_{1}\right)=0.7, \mu_{\tilde{R}}\left(x_{2}, y_{2}\right)=0.2, \mu_{\tilde{R}}\left(x_{2}, y_{3}\right)=0.3$

$$
\mu_{\tilde{R}}\left(x_{3}, y_{1}\right)=1, \mu_{\tilde{R}}\left(x_{3}, y_{2}\right)=0.6, \mu_{\tilde{R}}\left(x_{3}, y_{3}\right)=0.2
$$

\section{Example 2.4}

\begin{tabular}{|c|c|c|c|}
\hline \multirow{4}{*}{$\tilde{A} \times \tilde{B}=\tilde{R}=$} & $\mathrm{X}$ & $y_{1}$ & $y_{2}$ \\
\hline & $x_{1}$ & 0.2 & 0.2 \\
\hline & $x_{2}$ & 0.3 & 0.5 \\
\hline & $x_{3}$ & 0.3 & 0.9 \\
\hline
\end{tabular}

Assume two fuzzy sets

$\tilde{A}=\left\{\frac{0.2}{x_{1}}+\frac{0.5}{x_{2}}+\frac{1}{x_{3}}\right\}, \quad \tilde{B}=\left\{\frac{0.3}{y_{1}}+\frac{0.9}{y_{2}}\right\}$.

Find the fuzzy relation (the Cartesian product).

Solution:

\section{THE BASIC OPERATIONS ON FUZZY RELATIONS}

Let $\widetilde{R}_{1}$ and $\widetilde{R}_{2}$ be two fuzzy relations on $\widetilde{A} \mathrm{x} \widetilde{B}$ such that

$$
\begin{aligned}
& \widetilde{R}_{1}=\left\{(x, y), \mu_{\tilde{R}_{1}}(x, y)\right\},(x, y) \in \widetilde{A} \times \widetilde{B} \\
& \widetilde{R}_{2}=\left\{(x, y), \mu_{\tilde{R}_{2}}(x, y)\right\},(x, y) \in \widetilde{A} \times \widetilde{B}
\end{aligned}
$$

We use the membership functions $\mu_{\tilde{R}_{1}}(x, y)$ and $\mu_{\tilde{R}_{2}}(x, y)$ in order to introduce the operations with $\widetilde{R}_{1}$ and $\widetilde{R}_{2}$ similarly to operations with fuzzy sets.

\section{A. The equality}

$\widetilde{R}_{1}=\widetilde{R}_{2}$ ifffor $\quad$ every $\quad \operatorname{pair}(x, y) \in \widetilde{A} \mathrm{x} \widetilde{B}$, we have $\mu_{\tilde{R}_{1}}(x, y)=\mu_{\tilde{R}_{2}}(x, y)$.

\section{B. The inclusion}

The pair of $\operatorname{all}(x, y) \in \tilde{A} \times \tilde{B}$, then $\mu_{\tilde{R}_{1}}(x, y) \leq \mu_{\tilde{R}_{2}}(x, y)$, the

\begin{tabular}{|c|c|c|c|}
\hline$X$ & $y_{1}$ & $y_{2}$ & $y_{3}$ \\
\hline$x_{1}$ & 0 & 0.2 & 0.6 \\
\hline$x_{2}$ & 0.4 & 1.0 & 0.8 \\
\hline
\end{tabular}
relation $\widetilde{R}_{1}$ is included in $\widetilde{R}_{2}$ (or) $\widetilde{R}_{2}$ is larger than $\widetilde{R}_{1}$, denoted by $\tilde{R}_{1} \subseteq \tilde{R}_{2}$.

If $\widetilde{R}_{1} \subseteq \widetilde{R}_{2}$, in addition if for at least one pair $(x, y), \mu_{\tilde{R}_{1}}(x, y)<\mu_{\tilde{R}_{2}}(x, y)$, then we have the proper inclusion $\widetilde{R}_{1} \subset \widetilde{R}_{2}$.

\section{Example 3.1}

\begin{tabular}{|c|c|c|c|}
\hline$Y$ & $y_{1}$ & $y_{2}$ & $y_{3}$ \\
\hline$x_{1}$ & 0.1 & 0.2 & 0.7 \\
\hline$x_{2}$ & 0.5 & 1.0 & 0.9 \\
\hline
\end{tabular}

Published By:
\& Sciences Publication

(C) Copyright: All rights reserved. 
Then we have,

$$
\begin{aligned}
& \mu_{\tilde{R}_{1}}\left(x_{1}, y_{1}\right)=0<\mu_{\tilde{R}_{2}}\left(x_{1}, y_{1}\right)=0.1 \\
& \mu_{\tilde{R}_{1}}\left(x_{2}, y_{1}\right)=0.4<\mu_{\tilde{R}_{2}}\left(x_{2}, y_{1}\right)=0.5 \\
& \mu_{\tilde{R}_{1}}\left(x_{1}, y_{2}\right)=0.2=\mu_{\tilde{R}_{2}}\left(x_{1}, y_{2}\right)=0.2 \\
& \mu_{\tilde{R}_{1}}\left(x_{2}, y_{2}\right)=1.0=\mu_{\tilde{R}_{2}}\left(x_{2}, y_{2}\right)=1.0 \\
& \mu_{\tilde{R}_{1}}\left(x_{1}, y_{3}\right)=0.6<\mu_{\tilde{R}_{2}}\left(x_{1}, y_{3}\right)=0.7 \\
& \mu_{\tilde{R}_{1}}\left(x_{2}, y_{3}\right)=0.8<\mu_{\tilde{R}_{2}}\left(x_{2}, y_{3}\right)=0.9
\end{aligned}
$$

Hence $\widetilde{R}_{1}$ is included in $\widetilde{R}_{2}$, i.e. $\widetilde{R}_{1} \subset \widetilde{R}_{2}$.

\section{The complement}

The complement of a relation $\widetilde{R}$ denoted by $\widetilde{R}$, is defined by

$$
\mu_{\tilde{R}^{c}}(x, y)=1-\mu_{\tilde{R}}(x, y)
$$

\section{Example 3.2}

Consider the relation $\widetilde{R}$ is given by the table and find its complementation.

$\tilde{R}=$\begin{tabular}{|c|c|c|c|}
$Y$ & $y_{1}$ & $y_{2}$ & $y_{3}$ \\
\hline$x_{1}$ & 0 & 0.2 & 0.6 \\
\hline$x_{2}$ & 0.5 & 1.0 & 0.8 \\
\hline
\end{tabular}

Solution:

$\tilde{R}^{r}=$\begin{tabular}{|c|c|c|c|}
$Y$ & $y_{1}$ & $y_{2}$ & $y_{3}$ \\
\hline$x_{1}$ & 1.0 & 0.8 & 0.4 \\
\hline$x_{2}$ & 0.5 & 0 & 0.2 \\
\hline
\end{tabular}

\section{The union}

The union of $\widetilde{R}_{1}$ and $\widetilde{R}_{2}$ denoted by $\widetilde{R}_{1} \cup \widetilde{R}_{2}$, is defined by $\mu_{\tilde{R}_{1} \cup \tilde{R}_{2}}(x, y)=\max \left\{\mu_{\tilde{R}_{1}}(x, y), \mu_{\tilde{R}_{2}}(x, y)\right\},(x, y) \in \tilde{A} \times \tilde{B}$.

\section{E. The intersection}

The intersection of $\widetilde{R}_{1}$ and $\widetilde{R}_{2}$ denoted by $\widetilde{R}_{1} \cap \widetilde{R}_{2}$, is defined by

$\mu_{\tilde{R}_{1} \cap \tilde{R}_{2}}(x, y)=\min \left\{\mu_{\tilde{R}_{1}}(x, y), \mu_{\tilde{R}_{2}}(x, y)\right\},(x, y) \in \tilde{A} \mathrm{x} \tilde{B}$.

Example 3.3

The relations $\widetilde{R}_{1}$ and $\widetilde{R}_{2}$ are given by the following tables. Then calculate union and intersection.

$\tilde{R}_{1}=$\begin{tabular}{|c|c|c|c|}
\hline $\mathrm{Y}$ & $y_{1}$ & $y_{2}$ & $y_{3}$ \\
\hline$x_{1}$ & 0 & 0.1 & 0.2 \\
\hline$x_{2}$ & 0 & 0.7 & 0.3 \\
\hline$x_{3}$ & 0.2 & 0.8 & 1.0 \\
\hline
\end{tabular}

\begin{tabular}{|c|c|c|c|c|}
\hline \multirow{3}{*}{$\tilde{R}_{1} \cup \tilde{R}_{2}=$} & $x_{1}$ & 0.3 & 0.3 & 0.2 \\
\hline & $x_{2}$ & 0.5 & 0.7 & 1.0 \\
\hline & $x_{3}$ & 0.7 & 0.8 & 1 \\
\hline
\end{tabular}

$\tilde{R}_{2}=$\begin{tabular}{|c|c|c|c|}
\hline $\mathrm{Y}$ & $y_{1}$ & $y_{2}$ & $y_{3}$ \\
\hline$x_{1}$ & 0.3 & 0.3 & 0.2 \\
\hline$x_{2}$ & 0.5 & 0 & 1.0 \\
\hline$x_{3}$ & 0.7 & 0.3 & 0.1 \\
\hline
\end{tabular}

Solution:

(i).

(ii).

$\widetilde{R}_{1} \cap \widetilde{R}_{2}=$

\begin{tabular}{|c|c|c|c|}
\hline $\mathrm{Y}$ & $y_{1}$ & $y_{2}$ & $y_{3}$ \\
\hline$x_{1}$ & 0 & 0.1 & 0.2 \\
\hline$x_{2}$ & 0 & 0 & 0.3 \\
\hline$x_{3}$ & 0.2 & 0.3 & 0.1 \\
\hline
\end{tabular}

Obviously, the proper inclusion $\left(\widetilde{R}_{1} \cap \widetilde{R}_{2}\right) \subset\left(\tilde{R}_{1} \cup \widetilde{R}_{2}\right)$ holds.

\section{COMPOSITION OF FUZZY RELATIONS}

Let $\widetilde{R}$ isa fuzzy relation on the Cartesian space $\mathrm{XxY}$; $\widetilde{S}$ isa fuzzy relation on the Cartesian space YxZ; $\widetilde{T}$ isa fuzzy relation on the Cartesian space XxZ.

Therefore, fuzzy max-min and fuzzy max-product compositions are defined as $\tilde{T}=\widehat{R} o \tilde{S}$.

(i) Max-min composition

$$
\mu_{\tilde{T}}(x, z)=\bigcup_{y \in Y}\left\{\mu_{\tilde{R}}(x, y) \cap \mu_{\tilde{S}}(y, z)\right\}
$$

(ii)Max-product composition

$$
\mu_{\tilde{T}}(x, z)=\bigcup_{y \in Y}\left\{\mu_{\tilde{R}}(x, y) o \mu_{\tilde{S}}(y, z)\right\}
$$

\section{Example 4.1}

If $X=\left\{x_{1}, x_{2}\right\}, Y=\left\{y_{1}, y_{2}\right\}$, and $Z=\left\{z_{1}, z_{2}, z_{3}\right\}$.

Consider the following fuzzy relations:

$\begin{array}{llll}y_{1} & y_{2} z_{1} & z_{2} & z_{3}\end{array}$

$\tilde{R}=\begin{aligned} & x_{1} \\ & x_{2}\end{aligned}\left[\begin{array}{ll}0.7 & 0.5 \\ 0.8 & 0.4\end{array}\right]$ and $\tilde{S}=\begin{aligned} & y_{1} \\ & y_{2}\end{aligned}\left[\begin{array}{lll}0.9 & 0.6 & 0.5 \\ 0.1 & 0.7 & 0.5\end{array}\right]$

Find the max-min composition and max-product composition.

\section{Solution:}

(i)Using max-min composition,

$$
\begin{aligned}
& \mu_{\tilde{T}}\left(x_{1}, z_{1}\right)=\bigcup_{y \in Y}\left\{\mu_{\tilde{R}}\left(x_{1}, y\right) \cap \mu_{\tilde{S}}\left(y, z_{1}\right)\right\} \\
& =\max \{\min (0.7,0.9), \min (0.5,0.1)\} \\
& =\max \{0.7,0.1\}=0.7 \\
& z_{1} \quad z_{2} \quad z_{3}
\end{aligned}
$$

Therefore, $\quad \tilde{T}=\begin{aligned} & x_{1} \\ & x_{2}\end{aligned}\left[\begin{array}{lll}0.7 & 0.6 & 0.5 \\ 0.8 & 0.6 & 0.4\end{array}\right]$

\section{Published By:}

Blue Eyes Intelligence Engineering

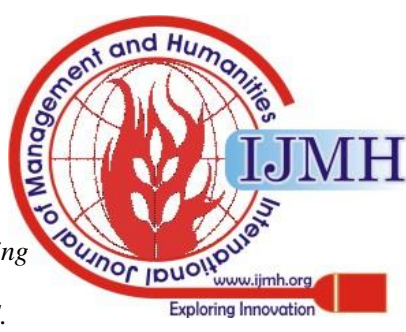




\section{Properties of Composition of Fuzzy Relations and its Verifications}

(ii) Using max-product composition,

$$
\begin{aligned}
& \mu_{\tilde{T}}\left(x_{1}, z_{2}\right)=\bigcup_{y \in Y}\left\{\mu_{\tilde{R}}\left(x_{1}, y\right) o \mu_{\tilde{S}}\left(y, z_{2}\right)\right\} \\
& =\max \{(0.7,0.6),(0.5,0.7)\} \\
& =\max \{0.42,0.35\}=0.42
\end{aligned}
$$

$$
\begin{array}{lll}
z_{1} & z_{2} & z_{3}
\end{array}
$$

Therefore, $\quad \tilde{T}=x_{x_{2}}^{x_{1}}\left[\begin{array}{lll}0.63 & 0.42 & 0.25 \\ 0.72 & 0.48 & 0.20\end{array}\right]$

\section{PROPERTIES OF COMPOSITION OF FUZZY RELATIONS}

Let $\widetilde{R}, \widetilde{R}_{1}, \widetilde{R}_{2}, \widetilde{R}_{3}$ are fuzzy relations. Then

(i) Composition of fuzzy with zero relation

$$
\tilde{R} \circ \tilde{O}=\tilde{O} \circ \tilde{R}(\tilde{O} \rightarrow \text { Zero Relation })
$$

(ii) Composition of fuzzy with identity relation

$$
\tilde{R} o \tilde{I}=\tilde{I} o \tilde{R}(\tilde{I} \rightarrow \text { Identity Relation })
$$

(iii) Composition of any two arbitrary fuzzy relations

$$
\widetilde{R}_{1} \circ \widetilde{R}_{2} \neq \widetilde{R}_{2} \circ \widetilde{R}_{1} \text { (In genral) }
$$

(iv)Composition of fuzzy subset relations

$\widetilde{R}_{2} \subseteq \widetilde{R}_{3} \Rightarrow\left(\widetilde{R}_{1} o \widetilde{R}_{2}\right) \subseteq\left(\widetilde{R}_{1} o \widetilde{R}_{3}\right)$ (In genral subset)

(v) Composition of arbitrary fuzzy associative relations $\widetilde{R}_{1} o\left(\widetilde{R}_{2} \circ \widetilde{R}_{3}\right)=\left(\widetilde{R}_{1} \circ \widetilde{R}_{2}\right) o \widetilde{R}_{3}$ (In genral associative)

(vi) Composition of arbitrary fuzzy distributive with union relations

\section{$\widetilde{R}_{1} \circ\left(\widetilde{R}_{2} \cup \widetilde{R}_{3}\right)=\left(\widetilde{R}_{1} \circ \widetilde{R}_{2}\right) \cup\left(\widetilde{R}_{1} \circ \widetilde{R}_{3}\right)$ (In genral union)}

(vii) Composition of arbitrary fuzzy distributive with intersection relations

(a) If all the elements in $\widetilde{R}_{2} \neq 0$ and either of atleast one element in both $\widetilde{R}_{1} \& \widetilde{R}_{3}$ are zeros (except for both non-zeros) or alternatively one is non-zero and other one is zero, then

$\widetilde{R}_{1} o\left(\widetilde{R}_{2} \cap \widetilde{R}_{3}\right)=\left(\widetilde{R}_{1} o \widetilde{R}_{2}\right) \cap\left(\widetilde{R}_{1} \circ \widetilde{R}_{3}\right)$ (In particular case)

(b) If all the elements in $\widetilde{R}_{2}=0$ and either of at least one element in both $\widetilde{R}_{1} \& \widetilde{R}_{3}$ are zeros or alternatively one is non-zero and other one is zero and also both of three relations in all the elements are non-zeros, then $\widetilde{R}_{1} o\left(\widetilde{R}_{2} \cap \widetilde{R}_{3}\right)=\left(\widetilde{R}_{1} o \widetilde{R}_{2}\right) \cap\left(\widetilde{R}_{1} o \widetilde{R}_{3}\right)$ (In particular case)

5.1 Verification of properties of composition of fuzzy relations

(i) $\tilde{R} \circ \tilde{O}=\tilde{O} \circ \tilde{R}$

Let $\widetilde{R}=\left[\begin{array}{ll}0.1 & 0.2 \\ 0.3 & 0.4\end{array}\right]$ and $\widetilde{O}=\left[\begin{array}{ll}0 & 0 \\ 0 & 0\end{array}\right]$

$\tilde{R} \circ \tilde{O}=\left[\begin{array}{ll}0.1 & 0.2 \\ 0.3 & 0.4\end{array}\right] \circ\left[\begin{array}{ll}0 & 0 \\ 0 & 0\end{array}\right]$

$=[\max \{\min (0.1,0), \min (0.2,0)\} \quad \max \{\min (0.1,0), \min (0.2,0)\}]$

$=\left[\begin{array}{ll}0 & 0 \\ 0 & 0\end{array}\right]$

$\widetilde{O} \circ \widetilde{R}=\left[\begin{array}{ll}0 & 0 \\ 0 & 0\end{array}\right] \circ\left[\begin{array}{ll}0.1 & 0.2 \\ 0.3 & 0.4\end{array}\right]$
Example 5.1

$=[\max \{\min (0.3,0), \min (0.4,0)\} \quad \max \{\min (0.3,0), \min (0.4,0)\}]$

$=\left[\begin{array}{ll}\max \{\min (0,0.1), \min (0,0.2)\} & \max \{\min (0.1,0), \min (0.2,0)\} \\ \max \{\min (0,0.3), \min (0,0.4)\} & \max \{\min (0,0.3), \min (0,0.4)\}\end{array}\right]$

$=\left[\begin{array}{ll}0 & 0 \\ 0 & 0\end{array}\right]$

$\therefore(1)=(2)$

(ii) $\tilde{R} \circ \tilde{I}=\tilde{I} o \tilde{R}$

Let $\widetilde{R}=\left[\begin{array}{ll}0.1 & 0.2 \\ 0.3 & 0.4\end{array}\right]$ and $\tilde{I}=\left[\begin{array}{ll}1 & 0 \\ 0 & 1\end{array}\right]$

$\tilde{R} o \hat{I}=\left[\begin{array}{ll}0.1 & 0.2 \\ 0.3 & 0.4\end{array}\right] \circ\left[\begin{array}{ll}1 & 0 \\ 0 & 1\end{array}\right]$

$=[\max \{\min (0.1,1), \min (0.2,0)\} \quad \max \{\min (0.1,0), \min (0.2,1)\}$

$=[\max \{\min (0.3,1), \min (0.4,0)\} \quad \max \{\min (0.3,0), \min (0.4,1)\}]$

$=\left[\begin{array}{ll}0.1 & 0.2 \\ 0.3 & 0.4\end{array}\right]$

$\tilde{I} o \tilde{R}=\left[\begin{array}{ll}1 & 0 \\ 0 & 1\end{array}\right] o\left[\begin{array}{ll}0.1 & 0.2 \\ 0.3 & 0.4\end{array}\right]$

$=\left[\begin{array}{ll}\max \{\min (1,0.1), \min (0,0.3)\} & \max \{\min (1,0.2), \min (0,0.4)\} \\ \max \{\min (0,0.1), \min (1,0.3)\} & \max \{\min (0,0.2), \min (1,0.4)\}\end{array}\right]$

$=\left[\begin{array}{ll}0.1 & 0.2 \\ 0.3 & 0.4\end{array}\right]$

$\therefore(1)=(2)$

(iii) $\widetilde{R}_{1} \circ \widetilde{R}_{2} \neq \widetilde{R}_{2} \circ \widetilde{R}_{1}$

Let $\quad \tilde{R}_{1}=\left[\begin{array}{ll}0.8 & 0.5 \\ 1.0 & 0.9\end{array}\right]$ and $\tilde{R}_{2}=\left[\begin{array}{ll}0.6 & 0.2 \\ 0.0 & 0.7\end{array}\right]$

$\widetilde{R}_{1} \circ \widetilde{R}_{2}=\left[\begin{array}{ll}0.8 & 0.5 \\ 1.0 & 0.9\end{array}\right] \circ\left[\begin{array}{ll}0.6 & 0.2 \\ 0.0 & 0.7\end{array}\right]$

$=[\max \{\min (0.8,0.6), \min (0.5,0.0)\} \quad \max \{\min (0.8,0.2), \min (0.5,0.7)\}$ $=\max \{\min (1.0,0.6), \min (0.9,0.0)\} \quad \max \{\min (1.0,0.2), \min (0.9,0.7)\}]$

$=\left[\begin{array}{ll}\max \{0.6,0.0\} & \max \{0.2,0.5\} \\ \max \{0.6,0.0\} & \max \{0.2,0.7\}\end{array}\right]$

$=\left[\begin{array}{ll}0.6 & 0.5 \\ 0.6 & 0.7\end{array}\right]$

$\widetilde{R}_{2} \circ \widetilde{R}_{1}=\left[\begin{array}{ll}0.6 & 0.2 \\ 0.0 & 0.7\end{array}\right] \circ\left[\begin{array}{ll}0.8 & 0.5 \\ 1.0 & 0.9\end{array}\right]$

$[\max \{\min (0.6,0.8), \min (0.2,1,0)\} \quad \max \{\min (0.6,0.5), \min (0.2,0.9)\}]$

$=[\max \{\min (0.0,0.8), \min (0.7,1.0)\} \quad \max \{\min (0.0,0.5), \min (0.7,0.9)\}]$

$=\left[\begin{array}{ll}\max \{0.6,0.2\} & \max \{0.5,0.2\} \\ \max \{0.0,0.7\} & \max \{0.0,0.7\}\end{array}\right]$

$=\left[\begin{array}{ll}0.6 & 0.5 \\ 0.7 & 0.7\end{array}\right]$

$\therefore(1) \neq(2)$

(iv) $\tilde{R}_{2} \subseteq \widetilde{R}_{3} \Rightarrow\left(\tilde{R}_{1} \circ \widetilde{R}_{2}\right) \subseteq\left(\widetilde{R}_{1} \circ \widetilde{R}_{3}\right)$

Let $\tilde{R}_{I}=\left[\begin{array}{ll}0.3 & 0.6 \\ 0.4 & 0.1\end{array}\right], \quad \tilde{R}_{2}=\left[\begin{array}{ll}0.2 & 0.5 \\ 0.0 & 0.8\end{array}\right]$ and $\tilde{R}_{3}=\left[\begin{array}{ll}0.4 & 0.7 \\ 0.1 & 0.8\end{array}\right]$

Given relations we conclude that, $\widetilde{R}_{2} \subseteq \widetilde{R}_{3}$.

Then $\quad \widetilde{R}_{1} \circ \widetilde{R}_{2}=\left[\begin{array}{ll}0.3 & 0.6 \\ 0.4 & 0.1\end{array}\right] \circ\left[\begin{array}{ll}0.2 & 0.5 \\ 0.0 & 0.8\end{array}\right]$

$[\max \{\min (0.3,0.2), \min (0.6,0.0)\} \quad \max \{\min (0.3,0.5), \min (0.6,0.8)\}]$

$=\max \{\min (0.4,0.2), \min (0.1,0.0)\} \max \{\min (0.4,0.5), \min (0.1,0.8)\}]$

$=[\max \{0.2,0.0\} \max \{0.3,0.6\}]$

$\max \{0.2,0.0\} \max \{0.4,0.1\}]$

$=\left[\begin{array}{ll}0.2 & 0.6 \\ 0.2 & 0.4\end{array}\right]$

Published By: 
open $\bigcirc$ Access

And $\quad \tilde{R}_{1} \circ \widetilde{R}_{3}=\left[\begin{array}{ll}0.3 & 0.0 \\ 0.4 & 0.1\end{array}\right] \circ\left[\begin{array}{ll}0.4 & 0.7 \\ 0.1 & 0.8\end{array}\right]$

$[\max \{\min (0.3,0.4), \min (0.6,0.1)\} \max \{\min (0.3,0.7), \min (0.6,0.8)\}]$

$=\max \{\min (0.4,0.4), \min (0.1,0.1)\} \max \{\min (0.4,0.7), \min (0.1,0.8)\}]$

$=[\max \{0.3,0.1\} \max \{0.3,0.6\}]$

$=[\max \{0.4,0.1\} \max \{0.4,0.1\}]$

$=\left[\begin{array}{ll}0.3 & 0.6 \\ 0.4 & 0.4\end{array}\right]$

From (1) \& (2) we have, $\left(\widetilde{R}_{l} \circ \widetilde{R}_{2}\right) \subseteq\left(\tilde{R}_{l} \circ \widetilde{R}_{3}\right)$.

(v) $\tilde{R}_{l} \circ\left(\widetilde{R}_{2} \circ \widetilde{R}_{3}\right)=\left(\widetilde{R}_{1} \circ \widetilde{R}_{2}\right) \circ \widetilde{R}_{3}$

Let $\tilde{R}_{I}=\left[\begin{array}{ll}0.7 & 0.2 \\ 0.4 & 0.8\end{array}\right], \quad \tilde{R}_{2}=\left[\begin{array}{ll}0.2 & 0.6 \\ 0.0 & 0.1\end{array}\right]$ and $\tilde{R}_{3}=\left[\begin{array}{ll}0.3 & 0.7 \\ 1.0 & 0.5\end{array}\right]$

$\widetilde{R}_{2} \circ \widetilde{R}_{3}=\left[\begin{array}{ll}0.2 & 0.6 \\ 0.0 & 0.1\end{array}\right] \circ\left[\begin{array}{ll}0.3 & 0.7 \\ 1.0 & 0.5\end{array}\right]$

$[\max \{\min (0.2,0.3), \min (0.6,1,0)\} \quad \max \{\min (0.2,0.7), \min (0.6,0.5)\}]$

$=\lfloor\max \{\min (0,0,0.3), \min (0.1,1,0)\} \max \{\min (0.0,0.7), \min (0.1,0.5)\}]$

$=[\max \{0.2,0.6\} \max \{0.2,0.5\}]$

$=[\max \{0.0,0.1\} \max \{0.0,0.1\}]$

$=\left[\begin{array}{ll}0.6 & 0.5 \\ 0.1 & 0.1\end{array}\right]$

$\widetilde{R}_{1} o\left(\widetilde{R}_{2} \circ \widetilde{R}_{3}\right)=\left[\begin{array}{ll}0.7 & 0.2 \\ 0.4 & 0.8\end{array}\right] \circ\left[\begin{array}{ll}0.6 & 0.5 \\ 0.1 & 0.1\end{array}\right]$

$[\max \{\min (0.7,0.6), \min (0.2,0.1)\} \max \{\min (0.7,0.5), \min (0.2,0.1)\}]$

$=\max \{\min (0.4,0.6), \min (0.8,0.1)\} \max \{\min (0.4,0.5), \min (0.8,0.1)\}]$

$=[\max \{0.6,0.2\} \max \{0.5,0.2\}]$

$=[\max \{0.4,0.1\} \max \{0.4,0.1\}]$

$=\left[\begin{array}{ll}0.6 & 0.5 \\ 0.4 & 0.4\end{array}\right]$

$\widetilde{R}_{1} o \widetilde{R}_{2}=\left[\begin{array}{ll}0.7 & 0.2 \\ 0.4 & 0.8\end{array}\right] \circ\left[\begin{array}{ll}0.2 & 0.0 \\ 0.0 & 0.1\end{array}\right]$

$[\max \{\min (0.7,0.2), \min (0.2,0.0)\} \quad \max \{\min (0.7,0.6), \min (0.2,0.1)\}]$

$=\max \{\min (0.4,0.2), \min (0.8,0.0)\} \max \{\min (0.4,0.6), \min (0.8,0.1)\}]$

$=[\max \{0.2,0.0\} \max \{0.6,0.1\}]$

$=[\max \{0.2,0.0\} \max \{0.4,0.1\}]$

$=\left[\begin{array}{ll}0.2 & 0.0 \\ 0.2 & 0.4\end{array}\right]$

$\left(\tilde{R}_{1} \circ \tilde{R}_{2}\right) \circ \tilde{R}_{3}=\left[\begin{array}{ll}0.2 & 0.6 \\ 0.2 & 0.4\end{array}\right] \circ\left[\begin{array}{ll}0.3 & 0.7 \\ 1.0 & 0.5\end{array}\right]$

$[\max \{\min (0.2,0.3), \min (0.6,1,0)\} \max \{\min (0.2,0.7), \min (0.6,0.5)\}]$

$=\max \{\min (0.2,0.3), \min (0.4,1,0)\} \max \{\min (0.2,0.7), \min (0.4,0.5)\}]$

$=[\max \{0.2,0.6\} \max \{0.2,0.5\}]$

$=[\max \{0.2,0.4\} \max \{0.2,0.4\}]$

$=\left[\begin{array}{ll}0.6 & 0.5 \\ 0.4 & 0.4\end{array}\right]$

$\therefore(2)=(4)$

(vi) $\quad \widetilde{R}_{1} o\left(\widetilde{R}_{2} \cup \widetilde{R}_{3}\right)=\left(\widetilde{R}_{1} o \widetilde{R}_{2}\right) \cup\left(\tilde{R}_{1} \circ \widetilde{R}_{3}\right)$

Let $\tilde{R}_{I}=\left[\begin{array}{ll}0.8 & 0.5 \\ 1.0 & 0.9\end{array}\right], \quad \tilde{R}_{2}=\left[\begin{array}{ll}0.6 & 0.2 \\ 0.0 & 0.7\end{array}\right]$ and $\tilde{R}_{3}=\left[\begin{array}{ll}0.0 & 1.0 \\ 0.4 & 0.3\end{array}\right]$

$\tilde{R}_{2} \cup \tilde{R}_{3}=\left[\begin{array}{ll}0.6 & 0.2 \\ 0.0 & 0.7\end{array}\right] \cup\left[\begin{array}{ll}0.0 & 1.0 \\ 0.4 & 0.3\end{array}\right]$

$=[\max \{0.6,0.0\} \max \{0.2,1.0\}]$

$=[\max \{0.0,0.4\} \max \{0.7,0.3\}]$

$=\left[\begin{array}{ll}0.6 & 1.0 \\ 0.4 & 0.7\end{array}\right]$

$\tilde{R}_{1} o\left(\widetilde{R}_{2} \cup \widetilde{R}_{3}\right)=\left[\begin{array}{ll}0.8 & 0.5 \\ 1.0 & 0.9\end{array}\right] \circ\left[\begin{array}{ll}0.6 & 1.0 \\ 0.4 & 0.7\end{array}\right]$ $=[\max \{\min (0.8,0.6), \min (0.5,0.4)\} \max \{\min (0.8,1.0), \min (0.5,0.7)\}]$

$=[\max \{\min (1,0,0.6), \min (0.9,0.4)\} \max \{\min (1,0,1,0), \min (0.9,0.7)\}]$

$=[\max \{0.6,0.4\} \max \{0.8,0.5\}]$

$=[\max \{0.6,0.4\} \max \{1.0,0.7\}]$

$=\left[\begin{array}{ll}0.6 & 0.8 \\ 0.6 & 1.0\end{array}\right]$

$\tilde{R}_{I} \circ \tilde{R}_{2}=\left[\begin{array}{ll}0.8 & 0.5 \\ 1.0 & 0.9\end{array}\right] \circ\left[\begin{array}{ll}0.6 & 0.2 \\ 0.0 & 0.7\end{array}\right]$

$[\max \{\min (0.8,0.6), \min (0.5,0.0)\} \max \{\min (0.8,0.2), \min (0.5,0.7)\}]$

$=\max \{\min (1,0,0.6), \min (0,9,0.0)\} \quad \max \{\min (1,0,0.2), \min (0.9,0.7)\}]$ $=[\max \{0.6,0.0\} \max \{0.2,0.5\}]$

$=[\max \{0.6,0.0\} \quad \max \{0.2,0.7\}]$

$=\left[\begin{array}{ll}0.6 & 0.5 \\ 0.6 & 0.7\end{array}\right]$

$\widetilde{R}_{1} \circ \widetilde{R}_{3}=\left[\begin{array}{ll}0.8 & 0.5 \\ 1.0 & 0.9\end{array}\right] \circ\left[\begin{array}{ll}0.0 & 1.0 \\ 0.4 & 0.3\end{array}\right]$

$[\max \{\min (0.8,0.0), \min (0.5,0.4)\} \max \{\min (0.8,1,0), \min (0.5,0.3)\}]$

$=\max \{\min (1,0,0.0), \min (0,9,0.4)\} \quad \max \{\min (1,0,1,0), \min (0,9,0.3)\}]$

$=\left[\begin{array}{ll}\max \{0.0,0.4\} & \max \{0.8,0.3\} \\ \max \{0.0,0.4\} & \max \{1.0,0.3\}\end{array}\right]$

$=\left[\begin{array}{ll}0.4 & 0.8 \\ 0.4 & 1.0\end{array}\right]$

$\left(\widetilde{R}_{1} \circ \widetilde{R}_{2}\right) \cup\left(\widetilde{R}_{1} \circ \widetilde{R}_{3}\right)=\left[\begin{array}{ll}0.6 & 0.5 \\ 0.6 & 0.7\end{array}\right] \cup\left[\begin{array}{ll}0.4 & 0.8 \\ 0.4 & 1.0\end{array}\right]$

$=\left[\begin{array}{ll}\max \{0.6,0.4\} & \max \{0.5,0.8\} \\ \max \{0.6,0.4\} & \max \{0.7,1.0\}\end{array}\right]$

$=\left[\begin{array}{ll}0.6 & 0.8 \\ 0.6 & 1.0\end{array}\right]$

$\therefore(2)=(5)$

(vii) Case (a): $\tilde{R}_{l} o\left(\widetilde{R}_{2} \cap \widetilde{R}_{3}\right)=\left(\widetilde{R}_{l} o \widetilde{R}_{2}\right) \cap\left(\tilde{R}_{l} o \widetilde{R}_{3}\right)$

Let $\tilde{R}_{I}=\left[\begin{array}{ll}0.3 & 0.2 \\ 0.1 & 0.0\end{array}\right], \quad \tilde{R}_{2}=\left[\begin{array}{ll}0.4 & 0.5 \\ 0.6 & 0.7\end{array}\right]$ and $\widetilde{R}_{3}=\left[\begin{array}{cc}0.0 & 0.1 \\ 0.3 & 0.2\end{array}\right]$

$\widetilde{R}_{2} \cap \widetilde{R}_{3}=\left[\begin{array}{ll}0.4 & 0.5 \\ 0.6 & 0.7\end{array}\right] \cap\left[\begin{array}{ll}0.0 & 0.1 \\ 0.3 & 0.2\end{array}\right]$

$=\left[\begin{array}{ll}\min \{0.4,0.0\} & \min \{0.5,0.1\} \\ \min \{0.6,0.3\} & \min \{0.7,0.2\}\end{array}\right]$

$=\left[\begin{array}{ll}0.0 & 0.1 \\ 0.3 & 0.2\end{array}\right]$

$\widetilde{R}_{I} o\left(\widetilde{R}_{2} \cap \widetilde{R}_{3}\right)=\left[\begin{array}{ll}0.3 & 0.2 \\ 0.1 & 0.0\end{array}\right] o\left[\begin{array}{cc}0.0 & 0.1 \\ 0.3 & 0.2\end{array}\right]$

$[\max \{\min (0.3,0.0), \min (0.2,0.3)\} \max \{\min (0.3,0.1), \min (0.2,0.2)\}$

$=\max \{\min (0.1,0.0), \min (0.0,0.3)\} \max \{\min (0.1,0.1), \min (0.0,0.2)\}$

$=[\max \{0.0,0.2\} \max \{0.1,0.2\}]$

$=\max \{0.0,0.0\} \max \{0.1,0.0\}]$

$=\left[\begin{array}{ll}0.2 & 0.2 \\ 0.0 & 0.1\end{array}\right]$

$\widetilde{R}_{1} \circ \widetilde{R}_{2}=\left[\begin{array}{ll}0.3 & 0.2 \\ 0.1 & 0.0\end{array}\right] \circ\left[\begin{array}{ll}0.4 & 0.5 \\ 0.6 & 0.7\end{array}\right]$

$[\max \{\min (0.3,0.4), \min (0.2,0.6)\} \max \{\min (0.3,0.5), \min (0.2,0.7)\}]$

$=[\max \{\min (0.1,0.4), \min (0.0,0.6)\} \max \{\min (0.1,0.5), \min (0.0,0.7)\}]$

$=\left[\begin{array}{ll}\max \{0.3,0.2\} & \max \{0.3,0.2\} \\ \max \{0.1,0.0\} & \max \{0.1,0.0\}\end{array}\right]$

$=\left[\begin{array}{ll}0.3 & 0.3 \\ 0.1 & 0.1\end{array}\right]$

$\tilde{R}_{1} \circ \widetilde{R}_{3}=\left[\begin{array}{ll}0.3 & 0.2 \\ 0.1 & 0.0\end{array}\right] \circ\left[\begin{array}{ll}0.0 & 0.1 \\ 0.3 & 0.2\end{array}\right]$ 


\section{Properties of Composition of Fuzzy Relations and its Verifications}

$[\max \{\min (0.3,0.0), \min (0.2,0.3)\} \max \{\min (0.3,0.1), \min (0.2,0.2)\}\}$

$=\max \{\min (0.1,0.0), \min (0.0,0.3)\} \max \{\min (0.1,0.1), \min (0.0,0.2)\}]$

$=[\max \{0.0,0.2\} \max \{0.1,0.2\}]$

$=\left[\begin{array}{ll}0.2 & 0.2 \\ 0.0 & 0.1\end{array}\right]$

$\left(\widetilde{R}_{1} \circ \widetilde{R}_{2}\right) \cap\left(\widetilde{R}_{1} \circ \widetilde{R}_{3}\right)=\left[\begin{array}{ll}0.3 & 0.3 \\ 0.1 & 0.1\end{array}\right] \cap\left[\begin{array}{ll}0.2 & 0.2 \\ 0.0 & 0.1\end{array}\right]$

$=\left[\begin{array}{cc}\min \{0.3,0.2\} & \min \{0.3,0.2\} \\ \min \{0.1,0.0\} & \min \{0.1,0.1\}\end{array}\right]$

$=\left[\begin{array}{ll}0.2 & 0.2 \\ 0.0 & 0.1\end{array}\right]$

$\therefore(2)=(5)$

Case (b): $\widetilde{R}_{1} o\left(\widetilde{R}_{2} \cap \widetilde{R}_{3}\right) \neq\left(\widetilde{R}_{1} o \widetilde{R}_{2}\right) \cap\left(\widetilde{R}_{1} o \widetilde{R}_{3}\right)$

Let $\widetilde{R}_{1}=\left[\begin{array}{ll}0.1 & 0.3 \\ 0.4 & 0.8\end{array}\right], \quad \tilde{R}_{2}=\left[\begin{array}{ll}0.4 & 0.2 \\ 0.5 & 0.3\end{array}\right]$ and $\widetilde{R}_{3}=\left[\begin{array}{ll}0.9 & 1.0 \\ 0.3 & 0.2\end{array}\right]$

$\widetilde{R}_{2} \cap \widetilde{R}_{3}=\left[\begin{array}{ll}0.4 & 0.2 \\ 0.5 & 0.3\end{array}\right] \cap\left[\begin{array}{ll}0.9 & 1.0 \\ 0.3 & 0.2\end{array}\right]$

$=[\min \{0.4,0.9\} \min \{0.2,1.0\}]$

$=\left[\begin{array}{ll}\min \{0.5,0.3\} & \min \{0.3,0.2\}\end{array}\right]$

$=\left[\begin{array}{ll}0.4 & 0.2 \\ 0.3 & 0.2\end{array}\right]$

$\widetilde{R}_{1} o\left(\widetilde{R}_{2} \cap \widetilde{R}_{3}\right)=\left[\begin{array}{ll}0.1 & 0.3 \\ 0.4 & 0.8\end{array}\right] o\left[\begin{array}{ll}0.4 & 0.2 \\ 0.3 & 0.2\end{array}\right]$

$=[\max \{\min (0.1,0.4), \min (0.3,0.3)\} \max \{\min (0.1,0.2), \min (0.3,0.2)\}]$

$=[\max \{\min (0.4,0.4), \min (0.8,0.3)\} \max \{\min (0.4,0.2), \min (0.8,0.2)\}]$

$=[\max \{0.1,0.3\} \max \{0.1,0.2\}]$

$=[\max \{0.4,0.3\} \max \{0.2,0.2\}]$

$=\left[\begin{array}{ll}0.3 & 0.2 \\ 0.4 & 0.2\end{array}\right]$

$\widetilde{R}_{1} \circ \widetilde{R}_{2}=\left[\begin{array}{ll}0.1 & 0.3 \\ 0.4 & 0.8\end{array}\right] \circ\left[\begin{array}{ll}0.4 & 0.2 \\ 0.5 & 0.3\end{array}\right]$

$[\max \{\min (0.1,0.4), \min (0.3,0.5)\} \max \{\min (0.1,0.2), \min (0.3,0.3)\}]$

$=[\max \{\min (0.4,0.4), \min (0.8,0.5)\} \max \{\min (0.4,0.2), \min (0.8,0.3)\}]$

$=[\max \{0.1,0.3\} \max \{0.1,0.3\}]$

$=[\max \{0.4,0.5\} \quad \max \{0.2,0.3\}]$

$=\left[\begin{array}{ll}0.3 & 0.3 \\ 0.5 & 0.3\end{array}\right]$

$\widetilde{R}_{1} \circ \widetilde{R}_{3}=\left[\begin{array}{ll}0.1 & 0.3 \\ 0.4 & 0.8\end{array}\right] \circ\left[\begin{array}{ll}0.9 & 1.0 \\ 0.3 & 0.2\end{array}\right]$

$[\max \{\min (0.1,0.9), \min (0.3,0.3)\} \max \{\min (0.1,1,0), \min (0.3,0.2)\}]$

$=\max \{\min (0.4,0.9), \min (0.8,0.3)\} \max \{\min (0.4,1.0), \min (0.8,0.2)\}]$ $=\left[\begin{array}{ll}\max \{0.1,0.3\} & \max \{0.1,0.2\} \\ \max \{0.4,0.3\} & \max \{0.4,0.2\}\end{array}\right]$

$=\left[\begin{array}{ll}0.3 & 0.2 \\ 0.4 & 0.4\end{array}\right]$

$\left(\widetilde{R}_{1} o \widetilde{R}_{2}\right) \cap\left(\widetilde{R}_{1} o \widetilde{R}_{3}\right)=\left[\begin{array}{ll}0.3 & 0.3 \\ 0.5 & 0.3\end{array}\right] \cap\left[\begin{array}{ll}0.3 & 0.2 \\ 0.4 & 0.4\end{array}\right]$

$=\left[\begin{array}{cc}\min \{0.3,0.3\} & \min \{0.3,0.2\} \\ \min \{0.5,0.4\} & \min \{0.3,0.4\}\end{array}\right]$

$=\left[\begin{array}{ll}0.3 & 0.2 \\ 0.4 & 0.3\end{array}\right]$

$\therefore(2) \neq(5)$

\section{Exercise Problems}

1. Verify $\widetilde{R}_{1} o\left(\widetilde{R}_{2} \cup \widetilde{R}_{3}\right)=\left(\widetilde{R}_{1} o \widetilde{R}_{2}\right) \cup\left(\widetilde{R}_{1} o \widetilde{R}_{3}\right)$.

$$
\text { If } \widetilde{R}_{1}=\left[\begin{array}{ll}
0.1 & 0.2 \\
0.3 & 0.4
\end{array}\right], \quad \widetilde{R}_{2}=\left[\begin{array}{ll}
0.0 & 0.1 \\
0.4 & 0.5
\end{array}\right] \text { and } \widetilde{R}_{3}=\left[\begin{array}{ll}
0.0 & 0.2 \\
0.5 & 0.6
\end{array}\right]
$$

Answer: $L H S=R H S=\left[\begin{array}{ll}0.2 & 0.2 \\ 0.4 & 0.4\end{array}\right]$

2. Verify $\widetilde{R}_{1} o\left(\widetilde{R}_{2} \cap \widetilde{R}_{3}\right)=\left(\widetilde{R}_{1} \circ \widetilde{R}_{2}\right) \cap\left(\widetilde{R}_{1} \circ \widetilde{R}_{3}\right)$. $=[\max \{0.0,0.0\} \max \{0.1,0.0\}]$

$$
\text { If } \widetilde{R}_{I}=\left[\begin{array}{ll}
0.2 & 0.0 \\
0.3 & 0.4
\end{array}\right], \quad \widetilde{R}_{2}=\left[\begin{array}{ll}
0.6 & 0.3 \\
0.1 & 0.8
\end{array}\right] \text { and } \widetilde{R}_{3}=\left[\begin{array}{cc}
0.4 & 0.7 \\
1.0 & 0.3
\end{array}\right]
$$

Answer: $L H S=R H S=\left[\begin{array}{ll}0.2 & 0.2 \\ 0.3 & 0.3\end{array}\right]$

3. Verify $\widetilde{R}_{1} o\left(\widetilde{R}_{2} \cap \widetilde{R}_{3}\right)=\left(\widetilde{R}_{1} o \widetilde{R}_{2}\right) \cap\left(\widetilde{R}_{1} o \widetilde{R}_{3}\right)$.

$$
\text { If } \widetilde{R}_{I}=\left[\begin{array}{ll}
0.1 & 0.2 \\
0.3 & 0.5
\end{array}\right], \quad \widetilde{R}_{2}=\left[\begin{array}{ll}
0.7 & 0.4 \\
0.8 & 1.0
\end{array}\right] \text { and } \widetilde{R}_{3}=\left[\begin{array}{ll}
0.6 & 0.0 \\
0.3 & 0.7
\end{array}\right]
$$

Answer: $L H S=R H S=\left[\begin{array}{ll}0.2 & 0.2 \\ 0.3 & 0.5\end{array}\right]$

4. Verify $\widetilde{R}_{1} o\left(\widetilde{R}_{2} \cap \widetilde{R}_{3}\right) \neq\left(\widetilde{R}_{1} o \widetilde{R}_{2}\right) \cap\left(\widetilde{R}_{1} o \widetilde{R}_{3}\right)$.

$$
\text { If } \widetilde{R}_{1}=\left[\begin{array}{ll}
0.0 & 0.2 \\
0.6 & 0.7
\end{array}\right], \quad \widetilde{R}_{2}=\left[\begin{array}{ll}
0.4 & 0.0 \\
0.3 & 0.9
\end{array}\right] \text { and } \widetilde{R}_{3}=\left[\begin{array}{ll}
0.1 & 0.5 \\
0.8 & 0.3
\end{array}\right]
$$

Answer: $L H S=\left[\begin{array}{ll}0.2 & 0.2 \\ 0.3 & 0.3\end{array}\right] \neq\left[\begin{array}{ll}0.2 & 0.2 \\ 0.4 & 0.5\end{array}\right]=R H S$

5. Verify $\widetilde{R}_{1} o\left(\widetilde{R}_{2} \cap \widetilde{R}_{3}\right) \neq\left(\widetilde{R}_{1} o \widetilde{R}_{2}\right) \cap\left(\widetilde{R}_{1} o \widetilde{R}_{3}\right)$.

$$
\text { If } \widetilde{R}_{I}=\left[\begin{array}{ll}
0.2 & 0.3 \\
0.4 & 0.6
\end{array}\right], \quad \widetilde{R}_{2}=\left[\begin{array}{cc}
0.0 & 0.1 \\
0.3 & 0.7
\end{array}\right] \text { and } \widetilde{R}_{3}=\left[\begin{array}{cc}
0.4 & 0.6 \\
0.9 & 0.2
\end{array}\right]
$$

Answer: $L H S=\left[\begin{array}{ll}0.3 & 0.2 \\ 0.3 & 0.2\end{array}\right] \neq\left[\begin{array}{ll}0.3 & 0.2 \\ 0.3 & 0.4\end{array}\right]=R H S$

6. Verify $\widetilde{R}_{1} o\left(\widetilde{R}_{2} \cap \widetilde{R}_{3}\right) \neq\left(\widetilde{R}_{1} o \widetilde{R}_{2}\right) \cap\left(\widetilde{R}_{1} o \widetilde{R}_{3}\right)$.

$$
\text { If } \widetilde{R}_{I}=\left[\begin{array}{ll}
0.2 & 0.0 \\
0.3 & 0.4
\end{array}\right], \quad \widetilde{R}_{2}=\left[\begin{array}{ll}
0.0 & 0.5 \\
0.3 & 0.7
\end{array}\right] \text { and } \widetilde{R}_{3}=\left[\begin{array}{ll}
0.9 & 0.1 \\
0.0 & 0.3
\end{array}\right]
$$

Answer: $L H S=\left[\begin{array}{ll}0.0 & 0.1 \\ 0.0 & 0.3\end{array}\right] \neq\left[\begin{array}{ll}0.0 & 0.1 \\ 0.3 & 0.3\end{array}\right]=R H S$

7. Verify $\widetilde{R}_{1} o\left(\widetilde{R}_{2} \cap \widetilde{R}_{3}\right) \neq\left(\widetilde{R}_{1} o \widetilde{R}_{2}\right) \cap\left(\widetilde{R}_{1} o \widetilde{R}_{3}\right)$.

$$
\text { If } \widetilde{R}_{I}=\left[\begin{array}{ll}
0.8 & 0.5 \\
1.0 & 0.9
\end{array}\right], \quad \widetilde{R}_{2}=\left[\begin{array}{ll}
0.6 & 0.2 \\
0.0 & 0.7
\end{array}\right] \text { and } \widetilde{R}_{3}=\left[\begin{array}{ll}
0.0 & 1.0 \\
0.4 & 0.3
\end{array}\right]
$$

Answer: $L H S=\left[\begin{array}{ll}0.0 & 0.3 \\ 0.0 & 0.3\end{array}\right] \neq\left[\begin{array}{ll}0.4 & 0.5 \\ 0.4 & 0.7\end{array}\right]=R H S$

\section{CONCLUSION}

In this paper, we have seen some definitions and basic operations related on fuzzy relations. Next the composition of fuzzy relations are defined in two ways such as max-min composition and max-product composition with example. The max-min composition and max-product composition are two compositions of fuzzy relations which is being made use in this paper to introduce new properties of composition of fuzzy relations which we have verified by solving a suitable problems with the newly introduced properties and have given the solution for the problems.

\section{SCOPE OF PROPERTIES}

In future this properties can be used to extend $3 \times 3,4 \times 4$, ...... etc., order of matrix and also this properties can be used to work out the problems of non-square matrix.

\section{REFERENCES}

1. Bezdek, J.C. and Harris,J.D., Fuzzy partitions and relations, Fuzzy Sets and Systems, 1 (1978), 111-127.

2. Buckley, E.E., An introduction to fuzzy logic and fuzzy sets. Advances in Soft Computing Series, Springer-verlag, Berlin Heidelberg New York, 2002.

3. Cornelis, C, De Cock, M, and Kerre, E.E., Intuitionistic fuzzy rough sets at the crossroads of imperfect knowledge, Expert Syst., vol. 20, no. 5, pp. 260-269, 2003.

\section{Published By:}

Blue Eyes Intelligence Engineering \& Sciences Publication

(C) Copyright: All rights reserved.

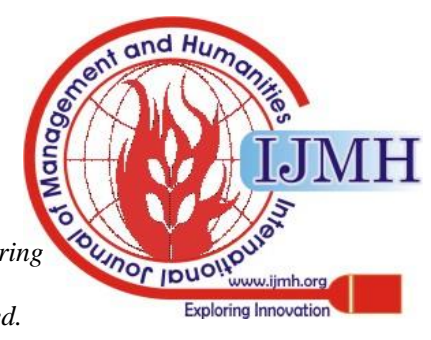


4. Dubios, D and Prade, H., Rough fuzzy sets and fuzzy rough sets, Int. J. Gen. Syst., vol. 17, pp.191-208, 1990.

5. George J.Klir, Tina A. Folger, Fuzzy Sets, Uncertainty, And Information published by Pearson Education, Inc, publishing as Prentice Hall (1988)

6. Gyanendra Kumar Panda, Surendra Prasad Jena, On the Theory of Fuzzy Relations, ISSN (Print):2395-7786, (Online):2395-7794, Volume-2,Issue-4,2016.

7. Nobuhara,H and Hirota,K., A Solution for Eigen Fuzzy Sets of Adjoint max-min composition and its Application to Image Analysis, IEEE, WISP, Budapest Hungary, vol. 2, pp. 27-30, Sept. 2003.

8. Ovchinnikov, S., An introduction to fuzzy relations, Fundamental of Fuzzy sets, The handbook of fuzzy sets series, Vol. 7 (2000), springer.

9. Sanchez,E., Eigen Fuzzy Sets Fuzzy Relations, Journal of Mathematical Analysis and Applications, vol. 81, pp.399-421, 1981.

10. Timothy J Ross, Fuzzy Logic with Engineering Applications, Third Edition (2010), John Wiley \& Sons, Ltd.

11. Zadeh,L.A., Fuzzy Sets, Inform.Control.8 (1965), 338-353.

12. Zimmermann, H.J., Fuzzy set theory and its applications, 2ndEd., Kluwer Academic Publishers (1991).

13. Zimmermann, H.J., Fuzzy relations and Fuzzy graphs, Fuzzy set theory and its applications, Springer Netherlands (2001), 71-91.

\section{AUTHORS PROFILE}

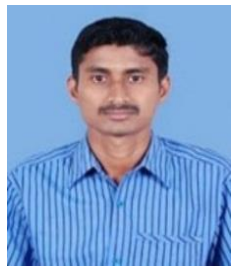

Mr. C. Gowrishankar, M.Sc.,M.Phil., B.Ed. is working as Assistant Professor for more than 6 years in the department of Mathematics at Government Arts \& Science College, Gudalur. He has also participated in several national \& international seminars. He has published two journals in IJMA Journal entitled 1). Comparitive study of HA, MOA and NAA methods for solving assignment problems, 2). Solving unbalanced assignment problem for using revised ones assignment method.

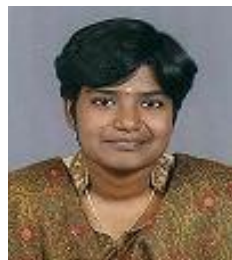

Miss. R. Dharshinee, M.Sc.,M.Phil. is working as Assistant Professor for more than 7 years in the department of Mathematics at Government Arts \& Science College, Gudalur. She has also participated in several national \& international seminars and published one journal in IJMA.

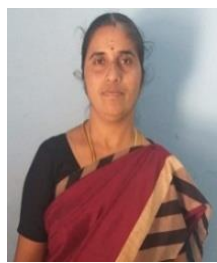

Mrs. K. Geetha, M.Sc.,M.Phil., B.Ed., SET. is working as Assistant Professor for more than 10 years in the department of Mathematics at Government Arts \& Science College, Gudalur. She has also participated in several national \& international seminars and published two journals.

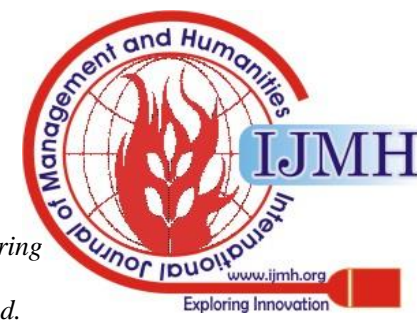

